Dominguez-Lara, Sergio*, a; Gravini-Donado, Marbel'; Torres-Villalobos, Guadalupe ${ }^{a}$

\title{
Artículo Metodológico
}

\section{Resumen}

\begin{abstract}
La Connor-Davidson Resilience Scale (CD-RISC) es una de las medidas de resiliencia a nivel mundial, pero no cuenta con evidencias de validez en Perú. EI objetivo del presente estudio fue analizar las propiedades psicométricas de la CD-RISC en estudiantes universitarios peruanos ( $n=746 ; 74.5 \%$ mujeres; $M_{\text {edad }}=19.99$ años). La estructura interna del CD-RISC fue analizada con análisis factorial confirmatorio y exploratory structural equation modeling (ESEM), así como su relación con medidas de autoeficacia y agotamiento emocional académico, y la confiabilidad del constructo y puntuaciones. Los resultados muestran que el modelo breve $y$ unidimensional de siete ítems (CD-RISC-7) recibe mayor respaldo considerando los índices de ajuste, magnitud de cargas factoriales, cumplimiento del supuesto de tau-equivalencia y ausencia de malas especificaciones. Asimismo, el CD-RISC-7 se asocia positiva y negativamente con autoeficacia académica y agotamiento emocional, respectivamente. Finalmente, sus indicadores de confiabilidad son meritorios. Se concluye que el CD-RISC-7 cuenta con propiedades psicométricas adecuadas.
\end{abstract}

Palabras clave:

ESEM, análisis factorial confirmatorio, resiliencia, universitarios

Recibido el 25 de marzo de 2019; Aceptado el 2 de julio de 2019

Editaron este artículo: Jazmín Cervasco, Paula Abate, Gabriela Rivarola, Yanina Michelini y Florencia Caneto

\section{Tabla de Contenido}

$\begin{array}{ll}\text { Introducción } & 36 \\ \text { Método } & 39 \\ \text { Participantes } & 39 \\ \text { Instrumentos } & 39 \\ \text { Procedimiento } & 40 \\ \text { Análisis de } & \\ \text { Datos } & 40 \\ \text { Resultados } & 41 \\ \text { Discusión } & 45 \\ \text { Referencias } & 47\end{array}$

\section{Introducción}

Durante la transición a la educación superior, los estudiantes viven experiencias tanto positivas como negativas que forman parte de la adaptación a esta nueva etapa. Estos eventos pueden ser de distinta índole: cambios en la metodología de enseñanza y formas de evaluar, nuevos compañeros y amistades, trasladarse de ciudad, asumir compromisos económicos, entre otros factores que pueden generar sentimientos de ansiedad, abandono o estrés académico. En este sentido, Pidgeon y Pickett, (2017) afirman que los estudiantes universitarios además de las exigencias académicas, enfrentan nuevos factores estresantes, como la necesidad de adaptarse a nuevas redes sociales, y que estas pueden tener un impacto negativo en su salud mental.

\footnotetext{
a Universidad de San Martín de Porres, Instituto de Investigación de Psicología, Lima, Perú.

b Universidad Simón Bolívar, Barranquilla, Colombia

"Enviar correspondencia a: Dominguez-Lara, S. E-mail: sdominguezl@usmp.pe
}

Citar este artículo como: Dominguez-Lara, S., Gravini-Donado, M., \& Torres-Villalobos, G. (2019). Análisis psicométrico de dos versiones de la Connor-Davidson Resilience Scale en estudiantes universitarios peruanos: propuesta del CD-RISC-7. Revista Argentina de Ciencias del Comportamiento, 11(2), 36-51 


\section{Dominguez-Lara, S. et al. / RACC, 2019, Vol. 11, №2, 36-51}

A su vez, a medida que avanzan los ciclos académicos, estarán sujetos a diferentes estresores vinculados con la vida académica, los cuales ponen a prueba su capacidad de enfrentarlo y alcanzar la meta de graduarse en una profesión u oficio. Por tanto, con el fin de prevenir fracasos académicos y personales, las instituciones de educación superior se ven apremiadas a realizar perfiles de ingreso de sus estudiantes para diseñar e implementar acciones que promuevan la permanencia y el bienestar estudiantil (Díaz Peralta, 2008; Pérez, Escobar, Toledo, Gutierrez, \& Reyes, 2018; Suárez-Montes \& Diaz-Subieta, 2015). De este modo, una característica personal se torna relevante: la resiliencia.

La resiliencia se define como una habilidad individual y medible para hacer frente y prosperar ante los diversos factores estresantes en que se pueda estar inmerso (Connor \& Davidson, 2003). Este constructo forma parte del conocimiento psicológico en diferentes ámbitos de aplicación desde hace décadas, y es así como en la educación superior los estudiantes atraviesan por una serie de exigencias académicas y personales para lograr su formación profesional que, a su vez, los pueden llevar a asumir conductas resilientes que involucran el optimismo, la valoración, el apoyo externo y las expectativas realistas conformando una comunidad universitaria más adaptativa (DeRosier, Frank, Schwartz, \& Leary, 2013; García \& Torbay, 2012). Esto puede verse reflejado en la tendencia de muchos jóvenes que cursan carreras profesionales y alcanzan puntajes medios y altos en las escalas de resiliencia general (Caldera, Aceves, \& Reynoso, 2016; García-León, González-Gómez, Robles-Ortega, Padilla, \& Peralta-Ramírez, 2019). Dichas características resilientes pueden indicar que estas valoraciones positivas sobre su capacidad de enfrentar el estrés redundarán en la búsqueda de las estrategias y recursos necesarios para permanecer y continuar con sus estudios (GraviniDonado, López, Marín-Escobar, \& Ortiz Padilla, 2017).

Asimismo, se ha observado que el grado de resiliencia guarda relación con algunas variables sociodemográficas o constructos que demuestran su característica protectora y moduladora de salud, como por ejemplo las variaciones del grado de resiliencia de acuerdo al nivel educativo, la correlación positiva con la personalidad resistente, el apoyo social, y las relaciones negativas con la depresión, ansiedad y el estrés (García-León et al., 2019), así como con el autoconcepto académico (Gravini-Donado, 2016).

En cuanto a variables psicológicas relevantes al contexto académico, se ha encontrado que la resiliencia se asocia positivamente con la autoeficacia académica (Bender \& Ingram, 2018; Cassidy, 2015; Li, Eschenauer, \& Persaud, 2018; Ríos-Risquez, Carrillo-García, \& Sabuco-Tebar, 2012; Ríos-Risquez, García-Izquierdo, SabucoTebar, Carrillo-Garcia, \& Martinez-Roche, 2016; Sameroff, \& Rosenblum, 2006; Vizoso-Gómez, \& Arias-Gundín, 2018) y negativamente con el agotamiento emocional académico (Ríos-Risquez et al., 2016; Vizoso-Gómez, \& Arias-Gundín, 2018) en el ámbito universitario. En ese sentido, un estudiante con creencias positivas acerca de su eficacia en los estudios podrá hacer frente a las dificultades, evitando el fracaso y adaptándose con éxito a los desafíos y exigencias del medio. Por otro lado, el agotamiento emocional aumenta cuando el individuo carece de factores de protección como la resiliencia, que le permitirán entender lo que está produciendo ese cansancio, lo que demanda de sí y cómo lo puede enfrentar.

Para llegar a estas conclusiones es necesario contar con escalas psicológicas estandarizadas, de rápida administración y de fácil acceso para una población numerosa teniendo en cuenta que la educación superior se ha masificado y que cada vez son más los estudiantes que ingresan con el deseo de ser profesionales y mejorar su calidad de vida. Sin embargo, el uso indiscriminado de escalas de medición psicológica sin evidencias de validez puede traer consecuencias indeseables (American Educational Research Association, American Psychological Association, \& National Council on Measurement in Education, 2014).

En ese sentido este artículo pretende aportar evidencias sobre las propiedades psicométricas de la Escala de Resiliencia de Connor y Davidson (CD-RISC; Connor \& Davidson, 2003) en población universitaria. EI CD-RISC es uno de los instrumentos más usados en la actualidad y que presenta una gran cantidad de estudios psicométricos a nivel mundial (e.g., Lee et al., 2013).

La CD-RISC evalúa la resiliencia como un constructo multidimensional con base en cinco dimensiones. La primera es la noción de 


\section{Dominguez-Lara, S. et al. / RACC, 2019, Vol. 11, N², 36-51}

competencia personal, autoexigencia $y$ tenacidad, que alude a tener certeza de la propia preparación para afrontar estresores (e.g., ítem 10: "Me esfuerzo al máximo en cada ocasión"); la confianza de la propia intuición y tolerancia a estímulos negativos, que trata de las expectativas positivas de las conductas individuales y la conformidad para sobrellevar la adversidad, aun habiendo otros estresores (e.g., ítem 7: "Afrontar el stress me fortalece"); también se encuentra la aceptación positiva al cambio y relaciones interpersonales seguras (e.g., ítem 8:"Tiendo a recuperarme de las enfermedades 0 de las dificultades") el cual refiere a la flexibilidad para adecuarse a distintas situaciones y establecer relaciones con personas confiables que den cabida a un crecimiento personal mutuo; el control, que consiste en acciones y cogniciones orientados al bienestar del individuo (e.g., ítem 21 "Siento que controlo mi vida"); y la influencia espiritual, que representa el valor que las personas otorgan a sus creencias religiosas o experiencias espirituales de encuentro consigo mismo (ítem 3 "Algunas veces dejo que el destino o Dios me ayude") (Connor \& Davidson, 2003).

No obstante, si bien esto podría representar una ventaja conceptual dado que brinda una amplitud de nociones para la interpretación de las puntuaciones obtenidas, a nivel empírico el panorama es diferente. Se han encontrado diferentes estructuras factoriales que van desde un factor hasta modelos jerárquicos multidimensionales con un factor de segundo orden (para una revisión ver García-León et al., 2019), por lo que aún no existe un consenso acerca de su estructura interna (Ponce-Cisternas, 2015).

Una revisión de las validaciones realizadas con población similar a la de este estudio indica que en India la estructura original no fue confirmada en estudiantes universitarios, encontrando una solución de cuatro factores, sin los ítems de la dimensión influencia espiritual (Singh \& Yu, 2010). Igualmente, en Estados Unidos (Madewell \& Ponce-Garcia, 2016) analizaron dos versiones del CD-RISC, tanto de 25 ítems como de 10 ítems (Campbell-Sills \& Stein, 2007), siendo esta última la que presentó mejor ajuste. De la misma manera, en Australia, el CD-RISC de 10 ítems se ajusta mejor como una escala unidimensional que la versión original de 25 ítems (Burns \& Anstey, 2010; Gucciardi,
Jackson, Coulter, \& Mallett, 2011). Por otro lado, en otros países no fue confirmada la estructura original, obteniéndose en su lugar una estructura de tres factores en Sudáfrica (Jorgensen \& Seedat, 2008), de cuatro factores en Irán (Sadat, 2009) o modelos alternativos de cinco factores en Corea del Sur (Baek, Lee, Joo, Lee, \& Choi, 2010).

La literatura disponible muestra estudios que respaldan la estructura factorial original de cinco factores (Gillespie, Chaboyer, \& Wallis, 2009), así como otros que no brindan evidencia a su favor. Por ejemplo, en España con una muestra de cuidadores no profesionales de personas dependientes mayores de 60 años, se propuso una versión de la CD-RISC de 21 ítems (Crespo, Fernández-Lansac, \& Soberón, 2014). Igualmente, en España se ha encontrado que el CD-RISC se configura por tres factores en una muestra de emprendedores (Manzano-García \& Ayala, 2013), mientras que en población general se confirmó una estructura unidimensional pero con baja varianza explicada (18.93\%; García-León et al., 2019). En otro contexto, Green et al. (2014) analizaron la CD-RISC en militares veteranos, descartando la estructura original y proponiendo un modelo de dos factores; y en universitarios también se confirmó la unidimensionalidad con enfoque ESEM (Ponce-Cisternas, 2015). Adicionalmente, en China (Fu, Leoutsakos, \& Underwood, 2014) y Turquía (Karairmak, 2010) tampoco pudieron recuperar la estructura original con sobrevivientes de terremotos, encontrando un modelo de dos y tres factores, respectivamente.

Una posible causa de esta diversidad de modelos es la variabilidad de poblaciones que participaron, ya que la resiliencia depende de las circunstancias, la cultura, la edad, y la personalidad (Richardson, 2002; Singh \& Yu, 2010), e incluso los eventos que han experimentado (e.g., Fu et al., 2014). Por otro lado, aunque la evidencia parece discordante en cuanto a la cantidad de dimensiones que configuran el CD-RISC, debe tenerse en cuenta además de las condiciones antes mencionadas, un aspecto clave que sirven para definir la estructura interna de un instrumento: la magnitud de las correlaciones interfactoriales.

En diversos estudios se han hallado correlaciones tan elevadas entre dimensiones, que la estructura parece aproximarse a la unidimensionalidad, aunque no fue discutida en 
los estudios de forma apropiada. Por ejemplo, en Green et al. (2014) las correlaciones entre los dos factores que proponen es de .80, y en determinados casos no hubo reporte de las correlaciones entre factores (Gillespie et al., 2009; Jorgensen \& Seedat, 2008; Lamond et al., 2008). Sin embargo, en algunos trabajos las correlaciones no fueron significativamente elevada por lo que la propuesta multidimensional posee mayor respaldo (Khoshouei, 2009; Singh \& Yu, 2010), mientras que en otros estudios la elevada correlación interfactorial se interpretó como indicio de redundancia y se optó por una estructura unidimensional (Burns \& Anstey, 2010).

Otro punto a considerar son los procedimientos que amenazan la validez de las conclusiones derivadas de los datos, como la combinación denominada Little Jiffy que consta de: a) análisis de componentes principales (ACP), donde no es posible diferenciar la proporción de varianza verdadera de la varianza específica; b) autovalor mayor que la unidad para determinar el número de factores, el cual llega a sobreestimar el número de factores necesarios para explicar la estructura de un instrumento; y c) rotación varimax, que fuerza la ortogonalidad (o independencia) de factores. Esta combinación de métodos no es recomendada para fines de análisis psicométrico (Lloret-Segura, FerreresTraver, Hernández-Baeza, \& Tomás-Marco, 2014; Watkins, 2018). En este sentido, se han identificado estudios que usaron el ACP para definir la dimensionalidad del CD-RISC (Gillespie et al., 2009; Lamond et al., 2008; Karairmak, 2010), y en otros donde se implementó completamente el Little Jiffy (Baek et al., 2010; Crespo et al., 2014; Manzano-García \& Ayala, 2013) lo que cuestiona la validez de los modelos factoriales hallados.

Con respecto a los estudios con muestras latinoamericanas, estos aún son escasos, aunque cabe destacar el estudio ejecutado con universitarios chilenos y españoles (PonceCisternas, 2015), donde se determinó la unidimensionalidad del CD-RISC en las dos muestras con uno de los procedimientos más recomendados en la actualidad: el modelamiento exploratorio de ecuaciones estructurales (ESEM; Asparouhov \& Muthén, 2009). Por otro lado, se encontró un estudio realizado en Colombia con la escala de 10 ítems corroborando su carácter unidimensional (Riveros, Bernal, Bohórquez,
Vinaccia, \& Quiceno, 2017).

En este orden de ideas, y pese a la cantidad de estudios instrumentales disponibles de otros países, no han sido hallados trabajos psicométricos del CD-RISC en universitarios peruanos, sea en su versión de 25 o de 10 ítems, por lo que es necesario contar con evidencias que permitan usarlos en este contexto.

Con base en lo expuesto previamente, el objetivo del presente trabajo fue analizar la estructura interna del CD-RISC en sus dos versiones y brindar información sobre la confiabilidad del constructo y de las puntuaciones, así como su relación con otras variables relacionadas al contexto académico, como agotamiento emocional académico y autoeficacia académica.

El estudio se justifica en la medida que el CDRISC es un instrumento que puede ser utilizado en el contexto universitario para detectar tempranamente estudiantes en riesgo de abandono y fracaso académico, debido a sus escasas competencias personales para ser resilientes frente a las adversidades que se pueden presentar en el ámbito académico o fuera de éste durante el transcurso de su carrera. Por ello, la medida de resiliencia puede ser un indicador de los sistemas de alerta temprana en la educación superior para emprender acciones que mitiguen problemáticas asociados con la adaptación universitaria y la deserción. De este modo, las dimensiones que evalúa dicha escala facilitan la exploración de habilidades personales de afrontamiento que pueden ser recursos para una intervención preventiva y mediante la modificación o el fortalecimiento de estas, lo que contrarrestaría los factores de riesgo inmersos, directa o indirectamente, en el alumno.

\section{Método}

\section{Participantes}

Fue evaluada una muestra intencional de 746 estudiantes universitarios (74.5\% mujeres; $25.5 \%$ varones) del primer al octavo ciclo de la carrera de psicología en una universidad de gestión privada ubicada en Lima Metropolitana. El rango de edad estuvo entre los 16 y 53 años $\left(M_{\text {edad }}=19.99\right.$; $D E_{\text {edad }}=3.103 ; 77.2 \%$ entre 18 y 26 años), $96 \%$ solteros y el $17.8 \%$ trabaja.

\section{Instrumentos}

Escala de Resiliencia de Connor y 
Davidson (CD-RISC; Connor \& Davidson, 2003). Es un instrumento de evaluación multidimensional de la resiliencia que está compuesto por 25 ítems en escala Likert con cinco opciones de respuesta desde en absoluto (0) hasta casi siempre (4). Todos los ítems se califican de forma directa: a más puntuación, mayor presencia del constructo evaluado. Fue utilizada la versión en español brindada por el autor original (Bobes et al., 2001).

Escala de Resiliencia de Connor y Davidson 10 (CD-RISC-10; Campbell-Sills \& Stein, 2007). Esta versión abreviada del CD-RISC cuenta con 10 ítems contenidos en la versión original, y presenta el mismo formato y opciones de respuesta.

$\begin{array}{ccc}\text { Escala de Autoeficacia } & \text { Percibida } \\ \text { Específica de Situaciones Académicas }\end{array}$
(EAPESA; Palenzuela, 1983). Se trata de una medida unidimensional de la autoeficacia académica formada por nueve ítems con cuatro opciones de respuesta (desde Nunca [1] hasta Siempre [4]). Todos los ítems se califican de forma directa: a más puntuación, mayor presencia del constructo evaluado. Fue usada la versión adaptada para universitarios peruanos que presenta evidencia favorable respecto a su unimensionalidad (Dominguez-Lara, 2016a).

Escala de Cansancio Emocional (ECE; Fontana, 2011). Se trata de una medida unidimensional del agotamiento emocional académico y está configurada por 10 ítems en formato Likert de cinco opciones (desde raras veces [1] hasta siempre [5]). Todos los ítems se califican de forma directa: a más puntuación, mayor presencia del constructo evaluado. Para el presente estudio se utilizó la versión adaptada a universitarios peruanos que posee evidencias de unidimensionalidad (Dominguez-Lara, FernándezArata, Manrique-Millones, Alarcón-Parco, \& DíazPeñaloza, 2018).

\section{Procedimiento}

El presente reporte fue desarrollado como parte del proyecto Relación entre burnout académico, ansiedad y depresión en estudiantes universitarios: análisis mediacional de factores protectores $y$ de riesgo aprobado por la universidad del autor corresponsal. Previo a la administración de los instrumentos, se solicitó el permiso correspondiente a las autoridades de la Facultad.
Los estudiantes fueron evaluados en el horario habitual de clases previa firma de un consentimiento informado. En dicho documento se indicaron los objetivos del estudio, además especificar que la participación era completamente voluntaria y que podían abandonar el proceso en cualquier momento. La investigación fue conducida bajo los principios de la declaración de Helsinki (Asociación Médica Mundial, 1964), así como del código de ética del Colegio de Psicólogos del Perú (2017).

\section{Análisis de datos}

Evidencias de validez con relación a la estructura interna del CD-RISC. Previo análisis descriptivo de los ítems, se realizó un análisis estructural de los modelos de medición disponibles para la versión de 25 ítems del CDRISC (desde un factor hasta cinco factores) vía CFA análisis factorial confirmatorio (CFA) con el método de extracción WLSMV con base en la matriz de correlaciones policóricas. De forma complementaria, se analizó la cantidad de malas especificaciones, es decir, la presencia o ausencia de parámetros que afectan la calidad del modelo (Saris, Satorra, \& Van der Veld, 2009), por medio de un módulo especializado (Dominguez-Lara \& Merino-Soto, 2018).

En el caso del ESEM, también se usó el método de extracción WLSMV solo con dos modelos: el original (cinco factores oblicuos) y un modelo bifactor (Rodriguez, Reise, \& Haviland, 2016a, 2016b) el cual postula que un solo factor general (FG) explica mayor variabilidad de los ítems en comparación a los factores específicos. Se especificó la rotación geomin $(\varepsilon=.05)$ para el modelo oblicuo y rotación target para el bifactor (Asparouhov, \& Muthen, 2009). En el modelo oblicuo fue interpretado el índice de simplicidad factorial (ISF; Fleming \& Merino, 2005) para analizar el impacto de las cargas factoriales secundarias, esperando valores mayores que .70 (simplicidad aceptable) que indicaría que el ítem recibe influencia significativa predominantemente de un factor. En cuanto al modelo bifactor, se analizó el coeficiente omega jerárquico general $\left(\omega_{h}\right)$ y de las dimensiones ( $\left.\omega_{h s}\right)$ (Zinbarg, Yovel, Revelle, \& McDonald, 2006), siendo este último sustancial cuando es $\geq .30$ (Smits, Timmerman, Barelds, \& Meijer, 2015), la varianza común explicada (ECV; Sijtsma, 2009) y el porcentaje de correlaciones no contaminadas por la 
multidimensionalidad (PUC; Reise, Scheines, Widaman, \& Haviland, 2013). Entonces, si el ECV y el PUC son mayores que .70, la varianza común puede considerarse unidimensional (Rodriguez et al., 2016a). Los cálculos fueron realizados con el módulo Índices Bifactor (Dominguez-Lara \& Rodriguez, 2017). Por último, el ajuste estadístico de los modelos se valoró según el índice de ajuste comparativo (CFI > .90; McDonald \& Ho, 2002), el límite superior del intervalo de confianza (IC) del índice de aproximación de la raíz de cuadrados medios del error (RMSEA < .10; West, Taylor, \& Wu, 2012), y la raíz cuadrada media residual ponderada (WRMR < 1; DiStefano, Liu, Jiang, \& Shi, 2018). De forma complementaria se consideró la magnitud de las cargas factoriales (> .50; Dominguez-Lara, 2018) y de las correlaciones interfactoriales $(\phi<.80$; Arias, 2008).

Evidencias de validez con relación a la estructura interna del CD-RISC-10. La versión de 10 ítems del CD-RISC fue analizada bajo un modelo unidimensional siguiendo los procedimientos establecidos anteriormente (WLSMV con base en matrices policóricas), y la valoración de los índices de ajuste y cargas factoriales fue la misma que para la versión extensa.

Todos los análisis basados en CFA y ESEM se llevaron a cabo con el programa Mplus versión 7 (Muthen \& Muthen, 1998-2015).

Confiabilidad del CD-RISC-7. Previo a su estimación, se evaluó la equivalencia estadística de las cargas factoriales de los ítems (tauequivalencia) de la versión de 10 ítems para justificar el uso del coeficiente a para la confiabilidad de las puntuaciones (DominguezLara, 2016b). Para ello, se compararon los índices de ajuste del modelo sin restricciones (modelo congenérico) y el que se restringió la igualdad de cargas (modelo tau-equivalente), y se rechaza la tau-equivalencia si $\Delta$ CFI $<-.01$ y $\triangle$ RMSEA $\geq .01$ (Chen, 2007). El coeficiente a fue complementado con sus IC (Dominguez-Lara, 2016c). Además, se consideró la correlación inter-ítem promedio $\left(r_{i j}\right)$ como medida alternativa de confiabilidad, esperando magnitudes entre .40 y .50 dado que se trata de un constructo esencialmente unidimensional (Clark \& Watson, 1995). Posteriormente, la confiabilidad del constructo se evaluó con el coeficiente $\omega(>.70$; Hunsley \& Marsh, 2008), y por último, la replicabilidad del constructo, que mide la máxima proporción de varianza de los ítems explicada por el factor, se evaluó con el coeficiente H (Rodriguez et al., 2016a),

Evidencias de equivalencia entre el CDRISC y CD-RISC-7. En este punto, fue considerada la correlación lineal entre ambas medidas como evidencia de equivalencia, pero ante la presencia de ítems comunes la correlación fue corregida (Bashaw \& Anderson, 1967; Levy, 1967). Luego, la magnitud de la diferencia entre ambas correlaciones (con y sin corrección) fue calculada con el estadístico q (Cohen, 1992): $\leq$ .10: trivial; > .10: pequeño; > .30: moderado; > .50: grande. Asimismo, los coeficientes $\alpha$ de ambas versiones fueron comparados estadísticamente con el programa AlphaTest (Lautenschlager \& Meade, 2008).

Evidencias de validez de la relación del CDRISC7 con otras variables. Se realizó un análisis estructural conjunto de los ítems de la EAPESA, ECE y CD-RISC-7 mediante un ESEM con rotación geomin, complementando con el ISF.

\section{Resultados}

\section{Análisis descriptivos}

La mayoría parte de los ítems presentan patrones de respuesta hacia la opción más elevada, evidenciando a su vez magnitudes leves de asimetría y curtosis.

Tabla 1.

Análisis descriptivo de los ítems del CD-RISC

\begin{tabular}{|c|c|c|c|c|}
\hline & $M$ & $D E$ & $g_{1}$ & $g_{2}$ \\
\hline Ítem 1 & 3.123 & 0.917 & -0.791 & -0.051 \\
\hline Ítem $2^{*}$ & 2.850 & 0.817 & -0.325 & -0.109 \\
\hline Ítem 3 & 2.013 & 1.126 & -0.038 & -0.698 \\
\hline Ítem 4* & 2.635 & 0.757 & -0.195 & 0.041 \\
\hline Ítem 5 & 3.066 & 0.853 & -0.842 & 0.787 \\
\hline Ítem $6^{*}$ & 2.999 & 0.838 & -0.492 & -0.261 \\
\hline Ítem $7^{*}$ & 2.634 & 0.938 & -0.515 & 0.148 \\
\hline Ítem 8* & 2.933 & 0.821 & -0.517 & -0.006 \\
\hline Ítem 9 & 3.188 & 0.878 & -1.103 & 1.190 \\
\hline Ítem 10 & 2.806 & 0.766 & -0.104 & -0.407 \\
\hline Ítem 11* & 3.133 & 0.753 & -0.583 & 0.213 \\
\hline Ítem 12 & 2.531 & 0.971 & -0.361 & -0.275 \\
\hline Ítem 13 & 2.724 & 1.050 & -0.582 & -0.252 \\
\hline Ítem $14^{*}$ & 2.198 & 1.107 & -0.243 & -0.538 \\
\hline Ítem 15 & 2.714 & 0.914 & -0.336 & -0.288 \\
\hline Ítem $16^{*}$ & 2.487 & 0.994 & -0.567 & 0.055 \\
\hline Ítem $17^{*}$ & 2.958 & 0.934 & -0.869 & 0.583 \\
\hline Ítem 18 & 2.525 & 0.891 & -0.208 & -0.050 \\
\hline Ítem 19* & 2.539 & 0.963 & -0.440 & -0.136 \\
\hline Ítem 20 & 2.421 & 0.933 & -0.252 & -0.157 \\
\hline Ítem 21 & 3.080 & 0.900 & -0.791 & 0.238 \\
\hline Ítem 22 & 2.729 & 0.892 & -0.498 & 0.039 \\
\hline Ítem 23 & 2.824 & 0.893 & -0.522 & 0.131 \\
\hline Ítem 24 & 3.029 & 0.789 & -0.612 & 0.458 \\
\hline Ítem 25 & 3.169 & 0.855 & -0.886 & 0.497 \\
\hline
\end{tabular}

Nota. *: ítems del CD-RISC-10; $g_{1}$ : asimetría; $g_{2}$ : curtosis 
Evidencias de validez con relación a la estructura interna del CD-RISC y CD-RISC-10

Como se puede apreciar, los índices de ajuste son adecuados en cuanto al CFI y RMSEA (excepto M8), aunque los valores del WRMR son elevados en todos los modelos. Sin embargo, un aspecto común son las correlaciones interfactoriales de fuerte magnitud en la mayoría de los modelos, llegando en varios casos a superar la unidad o ser elevadas (> .90; Tabla 2).

Tabla 2.

Índices de ajuste de los modelos de medición del CD-RISC

\begin{tabular}{|c|c|c|c|c|c|c|}
\hline Modelos & $N$ & CFI & RMSEA (IC 90\%) & WRMR & $\phi$ & $\phi_{\text {promedio }}$ \\
\hline & & & & & $\begin{array}{l}0.862,0.936 \\
0.927,0.902\end{array}$ & \\
\hline Original (M1) & 5 & .94 & $.061(.057, .065)$ & 1.345 & $\begin{array}{c}0.743,0.867 \\
0.294,0.298 \\
0.397,0.334 \\
0.879, .899 \\
0.749,1.057\end{array}$ & 0.656 \\
\hline Baek et al. (M2) & 5 & .95 & $.058(.054, .062)$ & 1.298 & $\begin{array}{l}\text { 0.977, 0.907, } \\
0.368,0.311 \\
0.360,0.370 \\
0.800,0.893 \\
0.893,0.912\end{array}$ & 0.688 \\
\hline Gillespie et al. (M3) & 5 & .96 & $.053(.049, .057)$ & 1.209 & $\begin{array}{l}0.782,0.906 \\
0.263,0.315 \\
0.373,0.335\end{array}$ & 0.647 \\
\hline Khoshouei (M4) & 4 & .93 & $.063(.059, .068)$ & 1.399 & $\begin{array}{c}1.067,1.094 \\
1.12,0.981 \\
0.983,1.067 \\
0.895,0.925\end{array}$ & 1.052 \\
\hline Lamond et al. (M5) & 4 & .94 & $.061(.057, .065)$ & 1.371 & $\begin{array}{l}0.999,0.304 \\
0.370,0.286\end{array}$ & 0.630 \\
\hline Singh \& Yu (M6) & 4 & .94 & $.062(.058, .066)$ & 1.386 & $\begin{array}{l}\text { 1.036, } 0.879 \\
0.940,0.856 \\
0.949,0.862\end{array}$ & 0.920 \\
\hline Jorgensen \& Seedat (M7) & 3 & .93 & $.064(.060, .068)$ & 1.443 & $\begin{array}{c}0.942,0.831 \\
0.679\end{array}$ & 0.817 \\
\hline Karairmak (M8) & 3 & .68 & $.139(.135, .142)$ & 3.086 & $\begin{array}{c}0.936,0.510 \\
0.571\end{array}$ & 0.672 \\
\hline Manzano-García \& Ayala (M9) & 3 & .93 & $.067(.063, .072)$ & 1.471 & $\begin{array}{c}0.937,0.996 \\
0.975\end{array}$ & 0.969 \\
\hline Fu et al. (M10) & 2 & .93 & $.066(.063, .070)$ & 1.492 & 1.047 & - \\
\hline Green et al. (M11) & 2 & .96 & $.074(.066, .081)$ & 1.257 & 0.856 & - \\
\hline Gucciardi et al. (M12) & 1 & .93 & $.066(.063, .070)$ & 1.495 & - & - \\
\hline
\end{tabular}

Nota. N: número de factores modelados; $\phi$ : correlación interfactorial

En vista de tal situación, se modelaron dos estructuras factoriales bajo enfoque ESEM, una oblicua (modelo original) y una bifactor. Tanto el modelo oblicuo (CFI $=.981$; RMSEA $=.041$ [.036, $.047]$, WRMR $=.720)$ como el bifactor $(\mathrm{CFI}=.987$; RMSEA $=.036[.031, .042]$, WRMR $=.617$ ) presentaron índices de ajuste favorables en todo sentido; sin embargo, es necesario resaltar algunas cosas.

En el modelo oblicuo no se aprecia una configuración sólida con respecto a la distribución de los ítems (Tabla 3). Por ejemplo, los ítems que originalmente pertenecen a competencia personal y tenacidad se distribuyen de forma irregular, o los ítems originales de algunos factores presentan tal complejidad que no logran agruparse satisfactoriamente (p. e., Control). Además, los ISF son muy bajos $(<.70)$ en gran parte de los casos (56\%; Tabla 3), pese a que las correlaciones interfactoriales fueron moderadas (Tabla 4), lo que indicaría que los ítems no son influidos predominantemente por su factor teórico, 
Dominguez-Lara, S. et al. / RACC, 2019, Vol. 11, N², 36-51

por lo que la presencia de un FG es plausible.

Tabla 3.

Análisis estructural del modelo oblicuo y bifactor vía ESEM

\begin{tabular}{|c|c|c|c|c|c|c|c|c|c|c|c|c|c|}
\hline & \multicolumn{6}{|c|}{ Modelo oblicuo } & \multicolumn{6}{|c|}{ Modelo bifactor } & \multirow{2}{*}{$\begin{array}{c}\text { Mod. } \\
\text { Unid. } \\
\text { F1 }\end{array}$} \\
\hline & F1 & F2 & F3 & F4 & F5 & ISF & FG & F1 & F2 & F3 & F4 & F5 & \\
\hline \multicolumn{14}{|l|}{ CPT } \\
\hline Ítem 24 & .139 & .159 & .128 & .599 & .033 & .817 & .673 & .407 & -.037 & -.030 & .144 & -.008 & .721 \\
\hline Ítem 11 & .075 & .216 & .402 & .347 & .025 & .477 & .754 & .201 & -.199 & .040 & .003 & -.024 & .751 \\
\hline Ítem 12 & .088 & .162 & .107 & .108 & .301 & .528 & .534 & -.021 & .124 & -.020 & .036 & -.048 & .538 \\
\hline Ítem 25 & .116 & .062 & .308 & .455 & .104 & .591 & .740 & .244 & -.160 & -.032 & .156 & .004 & .721 \\
\hline Ítem 10 & .138 & .078 & .167 & .511 & -.032 & .789 & .560 & .351 & -.174 & -.090 & .081 & .065 & .583 \\
\hline Ítem 23 & .112 & .430 & -.069 & .379 & .153 & .59 & .642 & .305 & .324 & .031 & .001 & -.111 & .682 \\
\hline Ítem 17 & -.008 & .149 & .290 & .045 & .526 & .678 & .747 & -.189 & .072 & -.073 & .075 & -.160 & .724 \\
\hline Ítem 16 & .090 & .178 & .266 & .001 & .414 & .555 & .695 & -.156 & .082 & -.018 & .017 & -.056 & .681 \\
\hline \multicolumn{14}{|l|}{ CSM } \\
\hline Ítem 20 & .395 & .033 & -.091 & -.019 & .176 & .755 & .292 & -.055 & .178 & -.026 & .040 & .283 & .305 \\
\hline Ítem 18 & .100 & .216 & -.024 & .022 & .437 & .730 & .530 & -.123 & .250 & -.083 & -.004 & -.055 & .534 \\
\hline Ítem 15 & .131 & .235 & .023 & .218 & .264 & .334 & .567 & .099 & .260 & .039 & .105 & -.064 & .600 \\
\hline Ítem 6 & .069 & .634 & .073 & -.121 & -.037 & .924 & .460 & -.013 & .209 & .141 & -.326 & -.033 & .454 \\
\hline Ítem 7 & .057 & .381 & .178 & -.070 & .194 & .586 & .565 & -.115 & .080 & .014 & -.197 & -.039 & .541 \\
\hline Ítem 19 & .182 & .153 & -.002 & -.020 & .509 & .782 & .572 & -.187 & .274 & -.100 & .061 & .010 & .576 \\
\hline Ítem 14 & .238 & .175 & -.093 & .054 & .297 & .423 & .432 & -.027 & .285 & -.052 & .032 & .085 & .453 \\
\hline \multicolumn{14}{|l|}{ RS } \\
\hline Ítem 1 & .237 & .135 & .571 & -.109 & -.130 & .706 & .486 & -.047 & -.074 & .741 & .100 & .099 & .495 \\
\hline Ítem 4 & .179 & .327 & .167 & .092 & .235 & .367 & .720 & -.016 & .059 & -.075 & -.136 & .061 & 699 \\
\hline Ítem 5 & .179 & .231 & .325 & .205 & .087 & .337 & .722 & .090 & -.094 & .037 & -.026 & .071 & .713 \\
\hline Ítem 2 & .181 & .476 & .110 & .107 & .015 & .756 & .597 & .133 & .212 & .162 & -.123 & .010 & .617 \\
\hline Ítem 8 & .141 & .220 & .317 & -.097 & .258 & .335 & .628 & -.173 & .019 & .048 & -.067 & .032 & .603 \\
\hline \multicolumn{14}{|l|}{ C } \\
\hline Ítem 22 & .195 & -.014 & .233 & .353 & .279 & .351 & .679 & .136 & .052 & .022 & .310 & .008 & .709 \\
\hline Ítem 13 & .205 & -.069 & .544 & .038 & .090 & .807 & .550 & -.014 & -.104 & .231 & .217 & .079 & .560 \\
\hline Ítem 21 & .140 & -.087 & .239 & .424 & .169 & .536 & .562 & .213 & -.054 & .011 & .326 & -.001 & .597 \\
\hline \multicolumn{14}{|l|}{ IE } \\
\hline Ítem 3 & .860 & -.139 & -.106 & -.125 & -.176 & .882 & .092 & .019 & .100 & .100 & .057 & .747 & .119 \\
\hline Ítem 9 & .348 & .049 & .124 & .016 & -.085 & .789 & .295 & .021 & -.113 & -.001 & -.070 & .336 & .285 \\
\hline
\end{tabular}

Nota. Mod. Unid.: Modelo unidimensional; CPT: Competencia personal y tenacidad; CSM: Confianza en sí mismo, tolerancia a las situaciones negativas y el fortalecimiento de los efectos del estrés; RS: Relaciones seguras y aceptación del cambio; C: Control; IE: Influencia espiritual; ISF: Índice de simplicidad factorial; En negrita: Cargas factoriales > .40; En cursiva: cargas factoriales pertenecientes a su factor original.

En cuanto al modelo bifactor, el FG presenta mayor fortaleza que los factores específicos considerando la magnitud de sus cargas factoriales $(84 \%$ tuvieron $\lambda>.45)$, ECV $=.779)$, PUC $=.790, \omega_{\mathrm{h}}=.906$, así como los omega jerárquico por dimensiones ( $\left.\omega_{\mathrm{hs}}\right)$ : Competencia personal y tenacidad (.039), Confianza en sí mismo, tolerancia a las situaciones negativas y el fortalecimiento de los efectos del estrés (.125), Relaciones seguras y aceptación del cambio 
(.063), Control (.130), e Influencia espiritual (.459). Entonces, la varianza de los ítems se explicaría mejor por la presencia de un FG que por cinco factores relacionados.

Posteriormente, la valoración del modelo unidimensional (M12) brinda resultados aceptables con respecto a sus índices de ajuste, aunque el WRMR es elevado, y buena confiabilidad de constructo ( $\omega=.927$; Tabla 3$)$. No obstante, existen 31 potenciales malas especificaciones asociadas a residuales correlacionados.

Tabla 4.

Correlaciones interfactoriales: modelo oblicuo ESEM.

\begin{tabular}{lccccc}
\hline & CPT & CSM & RS & C & IE \\
\hline CPT & 1 & & & & \\
CSM & .311 & 1 & & & \\
RS & .354 & .398 & 1 & & \\
C & .251 & .293 & .416 & 1 & \\
IE & .269 & .501 & .359 & .298 & 1 \\
\hline
\end{tabular}

Nota. CPT: Competencia personal y tenacidad; CSM: Confianza en sí mismo, tolerancia a las situaciones negativas y el fortalecimiento de los efectos del estrés; RS: Relaciones seguras y aceptación del cambio; C: Control; IE: Influencia espiritual.

Finalmente, la estructura unidimensional del CD-RISC-10 recibe respaldo empírico tanto a nivel de índices de ajuste (CFI $=.987$; RMSEA $=.043$ $[.032, .055]$, WRMR $=.740)$, cargas factoriales $(\approx$ .50; Tabla 5), y una cantidad mínima de malas especificaciones asociadas a residuales correlacionados $(n=3)$.

Tabla 5.

Análisis estructural y confiabilidad del CD-RISC-10

\begin{tabular}{lcc}
\hline & M1 & M2 \\
\hline İtem 2 & .625 & .614 \\
Ítem 4 & .721 & .713 \\
İtem 6 & .501 & - \\
İtem 7 & .582 & - \\
İtem 8 & .657 & .646 \\
İtem 11 & .674 & .689 \\
İtem 14 & .456 & - \\
İtem 16 & .718 & .725 \\
İtem 17 & .752 & .766 \\
İtem 19 & .611 & .606 \\
Confiabilidad & & \\
$\alpha$ (IC95\%) & - & .361 \\
$r_{i j}$ & - & .858 \\
$\omega$ & .870 & .864 \\
H & - & $.770, .823)$ \\
\hline
\end{tabular}

Nota. M1: CD-RISC-10; M2: Estructura modificada; rij: correlación inter-ítem promedio.

\section{Confiabilidad del CD-RISC-7}

Considerando los hallazgos previos acerca de la dimensionalidad del CD-RISC-10, se restringió la igualdad estadística de cargas factoriales (tauequivalencia) para justificar el uso del coeficiente $\alpha$. Si bien el ajuste fue aceptable (CFI = .930; RMSEA $=.091$ [.082, .101], WRMR $=1.890)$, la diferencia fue significativa respecto al modelo inicial $(\triangle \mathrm{CFI}=-.057 ; \triangle \mathrm{RMSEA}=.048)$, lo que no brinda respaldo suficiente a la tau-equivalencia. Así, en vista de la información brindada por los índices de modificación, fueron retirados del análisis tres ítems, siendo el ajuste del modelo unidimensional de estos siete ítems adecuados $(\mathrm{CFI}=.995$; RMSEA $=.038[.018, .057]$, WRMR = .535) y las diferencias menores con los índices de ajuste del modelo tau-equivalente $(\mathrm{CFI}=.980$; RMSEA $=.065[.051, .080], W R M R=1.201$ ) respaldan la igualdad estadística de las cargas factoriales $(\triangle \mathrm{CFI}=-.015 ; \triangle \mathrm{RMSEA}=.027)$. Con todo, el límite inferior del IC del coeficiente $\alpha$ de la versión de siete ítems fue de magnitud moderada (> .70; Tabla 5), mientras que los coeficientes $\omega$ y $\mathrm{H}$ fueron elevados (> .85; Tabla 5), y no presentó malas especificaciones.

\section{Evidencias de equivalencia entre el CD-RISC y CD-RISC-7}

La correlación entre las formas completa y breve fue elevada $(r=.888)$, considerando el valor mínimo recomendado para estos casos (> .70; Putnam \& Rothbart, 2006), y al corregir la correlación por la presencia de elementos comunes la magnitud se reduce $(r=.814)$, aunque siendo elevada y sin diferencias significativa respecto a la anterior $(q=.039)$. Con todo, el $\alpha$ de la versión extensa (.900) y la breve difieren de forma significativa $\left(\mathrm{X}^{2}{ }_{1}=232.856, p<.001\right)$; y la confiabilidad de constructo fue elevada en ambos casos $\left(\omega_{\text {CD-RISC }}=.927 ; \omega_{\text {CD-RISC-7 }}=.858\right)$.

\section{Evidencias de validez de la relación del CD- RISC-7 con otras variables}

Luego del modelamiento conjunto de los ítems del CD-RISC-7, ECE y EAPESA, además del ajuste óptimo logrado $(\mathrm{CFI}=.971$; $\mathrm{RMSEA}=.053$ $[.048, .057]$, WRMR $=.838)$, se aprecia que la distribución de los ítems de acuerdo con los constructos evaluados fue la esperada (Tabla 6), además que las cargas factores son de magnitud aceptable, y el ISF es elevado (excepto en el ítem 3 de agotamiento emocional). Estos aspectos previos respaldan los hallazgos que brindan evidencias de validez al CD-RISC-7: resiliencia presentó una asociación positiva con autoeficacia 
académica y negativa con agotamiento emocional.

Tabla 6.

Relación del CD-RISC con autoeficacia académica y agotamiento emocional.

\begin{tabular}{|c|c|c|c|c|}
\hline & $\mathbf{F 1}$ & F2 & F3 & ISF \\
\hline \multicolumn{5}{|l|}{ Resiliencia } \\
\hline$r 1$ & .604 & .025 & .054 & .986 \\
\hline r2 & .698 & .040 & .074 & .979 \\
\hline r3 & .678 & -.074 & -.094 & .955 \\
\hline r4 & .629 & .068 & .206 & .844 \\
\hline r5 & .706 & -.087 & .011 & .977 \\
\hline r6 & .786 & -.045 & -.040 & .991 \\
\hline$r 7$ & .691 & .061 & -.104 & .956 \\
\hline \multicolumn{5}{|l|}{$\begin{array}{l}\text { Agotamiento } \\
\text { emocional }\end{array}$} \\
\hline ae1 & -.127 & .587 & -.110 & .886 \\
\hline ae2 & -.211 & .485 & -.050 & .761 \\
\hline ae3 & -.391 & .486 & -.024 & .531 \\
\hline ae4 & .048 & .581 & .035 & .984 \\
\hline ae5 & -.164 & .627 & .000 & .906 \\
\hline ae6 & -.125 & .777 & .022 & .961 \\
\hline ae7 & -.122 & .775 & -.046 & .959 \\
\hline ae8 & -.065 & .725 & .031 & .985 \\
\hline ae9 & -.073 & .763 & -.102 & .961 \\
\hline ae10 & -.116 & .695 & -.100 & .931 \\
\hline \multicolumn{5}{|l|}{$\begin{array}{l}\text { Autoeficacia } \\
\text { académica }\end{array}$} \\
\hline au1 & .261 & -.075 & .701 & .810 \\
\hline au2 & .188 & -.085 & .715 & .886 \\
\hline au3 & .247 & -.076 & .749 & .844 \\
\hline au4 & .203 & -.070 & .693 & .871 \\
\hline au5 & .298 & -.104 & .623 & .707 \\
\hline au6 & .310 & -.043 & .706 & .766 \\
\hline aut & .231 & .022 & .634 & .829 \\
\hline au8 & .146 & -.030 & .725 & .940 \\
\hline au9 & .241 & .005 & .719 & .853 \\
\hline
\end{tabular}

Correlaciones

interfactoriales

\begin{tabular}{lccc} 
F1 & 1 & & \\
F2 & -.232 & 1 & \\
F3 & .381 & -.123 & 1 \\
Confiabilidad & & & \\
$\omega$ & .861 & .882 & .895 \\
\hline
\end{tabular}

Nota. r: resiliencia; ae: agotamiento emocional; au: autoeficacia académica; ISF: Índice de simplicidad factorial.

\section{Discusión}

El objetivo de la presente investigación fue realizar un análisis psicométrico de la CD-RISC en universitarios, considerando la necesidad de contar con una medida de resiliencia que pueda ser utilizada en el contexto de educación superior peruano debido la importancia del constructo en el desempeño social y académico del estudiante.

Dicho procedimiento se realizó en varias etapas, siendo la primera el análisis de su estructura interna. Fueron evaluados diversos modelos de medida bajo un enfoque CFA correspondientes a los distintos estudios revisados, y un aspecto común fue la presencia de elevadas correlaciones interfactoriales entre los primeros factores de cada modelo (e.g., si fueron cinco factores en un modelo, la asociación entre los cuatro iniciales fue más fuerte entre ellos que con el quinto factor). Este hallazgo específico tiene dos implicancias. La primera, que el último factor de cada modelo tiene menos aspectos compartidos con los demás factores al explicar la menor cantidad de varianza. La segunda, que un solo factor puede explicar la variabilidad de los ítems.

Así, los resultados derivados del ESEM refuerzan esas premisas: al modelar la estructura original de cinco factores existió una elevada complejidad factorial en muchos ítems, es decir, más de un factor influía simultánea y significativamente sobre ellos. Luego, al evaluar un modelo bifactor, el factor general modelado tiene más presencia que los factores específicos, excepto el último factor (influencia espiritual), pero es probable que se trate de un factor residual más que de uno sustantivo.

Estos resultados tienen escasa correspondencia con un elevado porcentaje de la literatura revisada, ya que algunos trabajos no consideraron el reporte o la interpretación de las correlaciones interfactoriales como indicios de redundancia factorial del CD-RISC y defienden la multidimensionalidad (e.g., Gillespie et al., 2009; Green et al., 2014; Jorgensen \& Seedat, 2008; Lamond et al., 2008) imposibilitando una interpretación más precisa de los datos. Inclusive, existen estudios que usaron métodos inadecuados para fines de análisis de estructura interna de un instrumento de evaluación psicológica (e.g., Baek et al., 2010; Crespo et al., 2014; Gillespie et al., 2009; Lamond et al., 2008; Karairmak, 2010; 


\section{Dominguez-Lara, S. et al. / RACC, 2019, Vol. 11, N², 36-51}

Manzano-García \& Ayala, 2013), muchos de los cuales enmascaran la correlación interfactorial con métodos que fuerzan la ortogonalidad de los componentes (e.g., rotación varimax en el paquete Little Jiffy) o extraen más factores de los necesarios (e.g., regla del autovalor mayor que uno). Esta situación trae consecuencias teóricas y prácticas, ya que interpretan de forma separada dimensiones que, empíricamente, no son distintas, puesto que desde la misma denominación a cada una de las dimensiones existen nociones teóricas interrelacionadas entre sí, y que además sus ítems reflejan cargas factoriales significativas en distintas dimensiones. Por el contrario, un número más reducido de estudios consideran métodos o interpretaciones más acordes con las asociaciones observadas entre dimensiones y concluyen a favor de la unidimensionalidad (Burns \& Anstey, 2010; Ponce-Cisternas, 2015).

De este modo, y en vista de la evidencia obtenida, se analizó un modelo unidimensional (e.g., los 25 ítems siendo influidos por una sola variable latente), obteniendo índices de ajuste adecuados y con solo dos ítems de carga factorial baja. Pese a ello, presentó un número significativo de malas especificaciones asociadas a residuales correlacionados, es decir, que fuentes ajenas al constructo podrían afectar la calidad del modelo (Saris et al., 2009). Entonces, si bien la CD-RISC mide un solo constructo con los 25 ítems, modelarlos de forma conjunta influye negativamente en el modelo. Por ese motivo, se consideró la versión breve de 10 ítems que, además de su practicidad y carácter unidimensional, presenta índices de ajuste adecuados y una cantidad casi nula de malas especificaciones, además de ser métricamente equivalente a la versión extensa. En este punto es necesario mencionar que no se han hallado estudios que aborden la equivalencia entre la versión original y las reducidas, por lo que los datos presentados en esta investigación podrían servir de referencia para futuros estudios similares.

Una vez definido el modelo a considerar, unidimensional y de 10 ítems, en la segunda etapa se evaluó la igualdad de cargas factoriales de los ítems previo al análisis de la confiabilidad. Si bien esto no es frecuente, es un paso necesario para justificar el uso del coeficiente $\alpha$, y de ese modo proponer estudios que más adelante puedan verificar la estabilidad de la estimación del error de medición (Green, 2003), o para garantizar la definición de puntos de corte confiables cuando se elaboren datos normativos (Livingston, 1972), cuyos cálculos se basan en la estimación del coeficiente $\alpha$. Entonces, aunque el modelo de 10 ítems es adecuado, los hallazgos indican que la tau-equivalencia se cumple solo al excluir tres ítems cuyas cargas factoriales difieren de los demás en magnitud, por lo que se consideró una versión final de siete enunciados. Esto se puede explicar con base en el contenido de los ítems, ya que los que fueron eliminados hacen referencia al afrontamiento en sí mismo (e.g., ítem 7: afrontar el estrés me fortalece), más que a la capacidad de recuperación frente a las adversidades o los cambios (e.g., ítem 2: soy capaz de adaptarme a los cambios), que es el punto central de la resiliencia. En ese sentido, la versión de siete ítems recoge el núcleo del constructo a evaluar.

La magnitud del coeficiente $\alpha$ hallado para los siete ítems si bien no permitiría decisiones a nivel individual debido a su magnitud $(\approx .80)$, pero desde un punto de vista de la asociación entre los ítems $\left(r_{i j}\right)$, su magnitud es consistente. Esto se refuerza con las magnitudes de los coeficientes enfocados en el constructo ( $\mathrm{H}$ y $\omega$ ), los que dan cuenta de la adecuada representación de la variable latente. Entonces, se puede concluir parcialmente que la confiabilidad de las puntuaciones y del constructo del CD-RISC-7 es adecuada.

La tercera etapa se enfocó en las evidencias de validez con relación a otras variables con el objetivo de brindar un marco conceptual de referencia a la CD-RISC. Entonces, acorde con los hallazgos, se determinó que la resiliencia se asocia positivamente con la autoeficacia académica y negativamente con el agotamiento emocional. En ese sentido, es evidente que el comportamiento del individuo y su capacidad de adaptación al entorno, no depende de manera exclusiva de una sola variable, y que la interdependencia de factores permitirá una mayor autorregulación emocional que le permitirá ser más eficaz y resiliente frente a las situaciones a las que se enfrenta para el logro de sus metas. Por ello, un estudiante que experimenta frustración, desinterés y estrés por las actividades académicas, puede presentar conductas de evitación o de afrontamiento que dependerán de sus características resilientes y de la percepción de sí mismo acerca de su autoeficacia, logrando 
superar los obstáculos propios de la vida académica o aquellos que son externos pero que inciden en su desempeño y bienestar en el ámbito universitario (Canales \& De los Ríos, 2009; Morales \& González, 2014; Villalta, 2010). De esta manera se asume la propuesta de un modelo en espiral entre la autoeficacia y el burnout, pero incluyendo el concepto de resiliencia en que el vínculo es positivo en relación a la variable de resiliencia y autoeficacia, y negativo entre el agotamiento emocional académico y la resiliencia (Salanova, Bresó, \& Schaufelli, 2005).

Si bien el objetivo del presente trabajo estuvo enfocado en la medida de resiliencia, más que en el constructo mismo, lo expuesto anteriormente evidencia la necesidad de seguir investigando teóricamente y empíricamente el concepto de resiliencia en el contexto peruano, teniendo en cuenta su complejidad y además considerando que la versión abreviada de la CD-RISC presenta óptimas propiedades psicométricas. Esto, porque la investigación sobre la resiliencia sigue siendo de amplio interés, toda vez que existen posturas teóricas en relación a si es una característica del ser humano (rasgo) o si es un proceso que sucede ante situaciones de estrés (estado) mediados por factores familiares, sociales e individuales (Luthar, Cicchetti, \& Becker, 2000).

Las implicancias prácticas de los resultados están directamente relacionadas con la posibilidad de incorporar la nueva medida (CDRISC-7) como parte de una caracterización de los estudiantes universitarios al inicio de su etapa académica con el fin de identificar aquellos estudiantes que presentan bajos puntajes de resiliencia y que por ende pueden ser susceptibles de abandono o fracaso. De la misma manera, se propone como un instrumento de evaluación para ser utilizado por los servicios de consejería psicológica que prestan los departamentos de bienestar estudiantil (o su equivalente en cada institución) a aquellos estudiantes que sean remitidos por presentar conductas disruptivas con el entorno y sus compañeros, bajo rendimiento académico o situaciones de crisis por eventos traumáticos familiares o individuales. Asimismo, la relevancia práctica de este resultado se encuentra en la posibilidad de utilizar el instrumento validado con población universitaria peruana, atendiendo a las propiedades psicométricas de la escala original.

Entre las limitaciones del estudio destaca la baja representatividad del género masculino, pese a ser lo esperado en carreras como psicología, de población predominantemente femenina. Aunado a ello, la aplicación a solo a estudiantes de psicología pudo sesgar los resultados hacia resultados más socialmente deseables, pero a juzgar por las medidas descriptivas halladas, las respuestas fueron consistentes y coherentes.

Con base en lo expuesto, se puede recomendar ampliar el rango de aplicación del instrumento, así como estudiarlo tanto en población de personas que han sufrido de traumas o adversidades como en la población en general, lo cual sería un buen indicio para profundizar en el debate respecto al estatus del constructo. En el plano de educación superior, sea técnica o universitaria, sería de interés analizar si el constructo resiliencia se asocia con variables vinculadas al éxito académico (promedio general, asignaturas desaprobadas, abandono, etc.), lo que podría dar mayor relevancia a su aplicación en ese ámbito. Asimismo, y desde un enfoque metodológico, sería conveniente analizar la estabilidad temporal del constructo mediante estudios de invarianza longitudinal que permitan cuantificar el error transitorio (Green, 2003), así como la invarianza de medición según variables sociodemográficas.

En conclusión, el CD-RISC-7 es una medida de resiliencia de carácter unidimensional que presenta evidencias de validez con relación a su estructura interna, incluyendo una cantidad mínima de malas especificaciones, y de relación con otras variables, así como indicadores satisfactorios de confiabilidad del constructo y de sus puntuaciones.

\section{Referencias}

American Educational Research Association, American Psychological Association, \& National Council on Measurement in Education. (2014). Standards for Educational and Psychological Testing. Washington, DC: American Educational Research Association.

Arias, B. (2008, Junio). Desarrollo de un ejemplo de análisis factorial confirmatorio con LISREL, AMOS y SAS. Trabajo presentado en el Seminario de Actualización en Investigación sobre Discapacidad, SAID. Universidad de Valladolid, España. Recuperado de: http://sid.usal.es/idocs/F8/FDO20749/said_2008.pd f 


\section{Dominguez-Lara, S. et al. / RACC, 2019, Vol. 11, №2, 36-51}

Asociación Médica Mundial (1964). Declaración de Helsinki. Helsinki: AMM. Recuperado de: http://www.conamed.gob.mx/prof_salud/pdf/helsink i.pdf

Asparouhov, T., \& Muthén, B. (2009). Exploratory structural equation modeling. Structural Equation Modeling, 16(3), 397-438. doi: $10.1080 / 10705510903008204$

Baek, H. S., Lee, K. U., Joo, E. J., Lee, M. Y., \& Choi, K. S. (2010). Reliability and Validity of the Korean Version of the Connor-Davidson Resilience Scale. Psychiatry Investigation, 7(2), 109-115. doi: 10.4306/pi.2010.7.2.109

Bashaw, W. L., \& Anderson, H. E. (1967). A correction for replicated error in correlation coefficients. Psychometrika, 32(4), 435-441.

Bender, A., \& Ingram, R. (2018). Connecting attachment style to resilience: Contributions of selfcare and self-efficacy. Personality and Individual Differences, 130, 18-20. doi: 10.1016/j.paid.2018.03.038

Bobes, J., Bascaran, M. T., García-Portilla, M. P., Bousoño, M., Sáiz, P. A., \& Wallance, D. H. (2001). Banco de instrumentos básicos de psiquiatría clínica. Barcelona: Psiquiatría ED.

Burns, R. A., \& Anstey, K. J. (2010). The Connor Davidson resilience scale (CD-RISC): Testing the invariance of a unidimensional resilience measure that is independent of positive and negative affect. Personality and Individual Differences, 48(5), 527531. doi: 10.1016/j.paid.2009.11.026

Caldera, J., Aceves, B., \& Reynoso, O. (2016). Resiliencia en estudiantes universitarios. Un estudio comparado entre carreras. Psicogente, 19(36), 227-239. doi: 10.17081/psico.19.36.1294

Campbell-Sills, L., \& Stein, M. (2007) Psychometric analysis and refinement of the Connor-Davidson resilience scale (CD-RISC): validation of a 10-item measure of resilience. Journal of Trauma Stress, 20(6), 1019-1028. doi: 10.1002/jts.20271

Canales, A., \& De los Ríos, D. (2009). Retención de estudiantes vulnerables en la educación universitaria. Calidad en la Educación, 30, 50-83.

Cassidy, S. (2015). Resilience building in students: the role of academic self-efficacy. Frontiers in Psychology, 6, 1781 . doi: 10.3389/fpsyg.2015.01781

Chen, F. F. (2007). Sensitivity of goodness of fit indexes to lack of measurement invariance. Structural Equation Modeling, 14(3), 464-504. doi: 10.1080/10705510701301834

Clark, L. A., \& Watson, D. (1995). Constructing validity: Basic issues in objective scale development. Psychological Assessment, 7(3), 309 - 319.

Cohen, J. (1992). A power primer. Psychological Bulletin, 112(1), 155-159. doi: 10.1037/00332909.112.1.155
Colegio de Psicólogos del Perú (2017). Código de ética y deontología. Recuperado de: https://www.cpsp.pe/documentos/marco_legal/codi go_de_etica_y_deontologia.pdf

Connor, K. M., \& Davidson, J. R. (2003). Development of a new resilience scale: the Connor Davidson Resilience Scale (CD-RISC). Depression and Anxiety, 18(2), 76-82. doi: 10.1037/t06346-000

Crespo, M., Fernández-Lansac, V., \& Soberón, C. (2014). Adaptación española de la escala de resiliencia de Connor-Davidson (CD-RISC) en situaciones de estrés crónico. Psicología Conductual, 22(2), 219-238.

DeRosier, M. E., Frank, E., Schwartz, V., \& Leary, K. A. (2013). The potential role of resilience education for preventing mental health problems for college students. Psychiatric Annals, 43(12), 538-544. doi: 10.3928/00485713-20131206-05

Díaz Peralta, C. (2008). Modelo conceptual para la deserción estudiantil universitaria chilena. Estudios Pedagógicos (Valdivia), 34(2), 65-86.doi: 10.4067/S0718-07052008000200004

DiStefano, C., Liu, J., Jiang, N., \& Shi, D. (2018). Examination of the weighted root mean square residual: Evidence for trustworthiness? Structural Equation Modeling: A Multidisciplinary Journal, 25(3), $\quad$ 453-466.

10.1080/10705511.2017.1390394

Dominguez-Lara, S. (2016a). Valores normativos de una escala de autoeficacia académica en estudiantes universitarios de Lima. Interacciones, 2(2), 91-98.

Dominguez-Lara S. (2016b). Secretos del coeficiente alfa. Actas Urológicas Españolas, 40(7), 471.

Dominguez-Lara, S. (2016c). Intervalos de confianza en el reporte de la fiabilidad: un análisis necesario. Anales del Sistema Sanitario de Navarra, 39(1), 169 - 170 . doi: 10.4321/S1137$6627 / 2016000100024$

Dominguez-Lara, S. (2018). Propuesta de puntos de corte para cargas factoriales: una perspectiva de fiabilidad de constructo. Enfermería Clínica, 28(6), 401-402. doi: 10.1016/j.enfcli.2018.06.002

Dominguez-Lara, S., Fernández-Arata, M., ManriqueMillones, D., Alarcón-Parco, D., \& Díaz-Peñaloza, M. (2018). Datos normativos de una escala de agotamiento emocional académico en estudiantes universitarios de psicología de Lima (Perú). Educación Médica, 19(3), 246 - 255. doi: 10.1016/j.edumed.2017.09.002

Dominguez-Lara, S., \& Merino-Soto, C. (2018). Análisis de las malas especificaciones en modelos de ecuaciones estructurales. Revista Argentina de Ciencias del Comportamiento, 10(2), 19-24. doi: 10.30882/1852.4206.v10.n2.19595

Dominguez-Lara, S., \& Rodriguez, A. (2017). Índices estadísticos de modelos bifactor. Interacciones, 


\section{Dominguez-Lara, S. et al. / RACC, 2019, Vol. 11, №2, 36-51}

3(2), 59-65. doi: 10.24016/2017.v3n2.51

Fleming, J., \& Merino, C. (2005). Medidas de simplicidad y ajuste factorial: Un enfoque para la construcción y revisión de escalas derivadas factorialmente. Revista de Psicología, 23(2), 252266.

Fontana, S. (2011). Estudio preliminar de las propiedades psicométricas de la escala de desgaste emocional para estudiantes universitarios. Revista Argentina de Ciencias del Comportamiento, 3(2), 44-48.

Fu, C., Leoutsakos, J. M., \& Underwood, C. (2014). An examination of resilience cross-culturally in child and adolescent survivors of the 2008 China earthquake using the Connor-Davidson Resilience Scale (CD-RISC). Journal of Affective Disorders, 155, 149-153. doi: 10.1016/j.jad.2013.10.041

García, M., \& Torbay, A. (2012). Universidad y Resiliencia, metamorfosis del potencial humano. En A. Fores, y J. Grané (Eds.), La Resiliencia en los entornos socioeducativos (pp. $22-25)$. Madrid: Narcea.

García-León, M. A., González-Gómez, A., RoblesOrtega, H., Padilla, J. L., \& Peralta-Ramírez, I. (2019). Psychometric properties of the ConnorDavidson Resilience Scale (CD-RISC) in the Spanish population. Anales de Psicología, 35(1), 33-40. doi: 10.6018/analesps.35.1.314111

Gillespie, B. M., Chaboyer, W., \& Wallis, M. (2009) The influence of personal characteristics on the resilience of operating room nurses: A predictor study. International Journal of Nursing Studies, 46(7), 968-976. doi: 10.1016/j.jijnurstu.2007.08.006

Gravini-Donado, M. (2016). La permanencia y la deserción estudiantil y su relación con el autoconcepto, la resiliencia y el rendimiento académico en estudiantes con riesgo socioeconómico alto o bajo (tesis de doctorado). Universidad Autónoma de Madrid, España.

Gravini-Donado, M., López, L., Marín-Escobar, J. C., \& Ortiz-Padilla, M. (2017). Dimensiones de la resiliencia en estudiantes desertores de la educación superior: Una mirada a sus posibilidades de reintegro. En M. Gravini-Donado, J. C. Marín-Escobar, y S. Falla (Comps.), Aportes a la calidad educativa desde la investigación (pp. 121 - 141). Barranquilla: Ediciones Universidad Simón Bolívar.

Green, S. B. (2003). A coefficient alpha for test-retest data. Psychological Methods, 8(1), 88-101. doi: 10.1037/1082-989X.8.1.88

Green, K. T., Hayward, L. C., Williams, A. M., Dennis, P. A., Bryan, B. C., Taber, K. H., \& Calhoun, P. S. (2014). Examining the factor structure of the Connor-Davidson resilience scale (CD-RISC) in a post-9/11 US military veteran sample. Assessment, 21(4), 443-451. doi: 10.1177/1073191114524014
Gucciardi, D. F., Jackson, B., Coulter, T. J., \& Mallett, C. J. (2011). The Connor-Davidson Resilience Scale (CD-RISC): Dimensionality and age-related measurement invariance with Australian cricketers. Psychology of Sport and Exercise, 12(4), 423-433. doi: 10.1016/j.psychsport.2011.02.005

Hunsley, J., \& Marsh, E. J. (2008). Developing criteria for evidence-based assessment: An introduction to assessment that work. En J. Hunsley \& E. J. Marsh (Eds.) A guide to assessments that work (pp. 3-14). Oxford: Oxford University Press.

Jorgensen, I., \& Seedat, S. (2008). Factor structure of the Connor-Davidson Resilience Scale in South African adolescents. International Journal of Adolescent Medicine and Health, 20(1), 23-32. doi: 10.1515/IJAMH.2008.20.1.23

Karairmak, Ö. (2010). Establishing the psychometric qualities of the Connor-Davidson Resilience Scale (CD-RISC) using exploratory and confirmatory factor analysis in a trauma survivor sample. Psychiatry Research, 179(3), 350-356. doi: 10.1016/j.psychres.2009.09.012

Khoshouei, M. S. (2009). Psychometric evaluation of the Connor-Davidson resilience scale (CD-RISC) using Iranian students. International Journal of Testing, 9(1), 60-66. doi: 10.1080/15305050902733471

Lamond, A. J., Depp, C. A., Allison, M., Langer, R., Reichstadt, J., Moore, D. J., \& Jeste, D. V. (2008). Measurement and predictors of resilience among community-dwelling older women. Journal of Psychiatric Research, 43(2), 148-154. doi: 10.1016/j.jpsychires.2008.03.007

Lautenschlager, G. J., \& Meade, A. W. (2008). AlphaTest: $A$ windows program for tests of hypotheses about coefficient alpha. Applied Psychological Measurement, 32(6), 502-503. doi: $10.1177 / 0146621607312307$

Lee, J. H., Nam, S. K., Kim, A. R., Kim, B., Lee, M. Y., \& Lee, S. M. (2013). Resilience: a meta-analytic approach. Journal of Counseling \& Development, 91(3), 269-279. doi: 10.1002/j.15566676.2013.00095.x

Levy, P. (1967). The correction for spurious correlation in the evaluation of short-form tests. Journal of Clinical Psychology, 23, 84-86.

Li, M., Eschenauer, R., \& Persaud, V. (2018). Between avoidance and problem solving: resilience, selfefficacy, and social support seeking. Journal of Counseling \& Development, 96(2), 132-143. doi: $10.1002 /$ jcad. 12187

Livingston, S. A. (1972). Criterion-referenced applications of classical test theory. Journal of Educational Measurement, 9(1), 13-26.

Lloret-Segura, S., Ferreres-Traver, A., HernándezBaeza, A., \& Tomás-Marco, I. (2014). El análisis factorial exploratorio de los ítems: una guía 


\section{Dominguez-Lara, S. et al. / RACC, 2019, Vol. 11, №2, 36-51}

práctica, revisada y actualizada. Anales de Psicología, 30(3), 1151-1169. doi: 10.6018/analesps.30.3.199361

Luthar, S. S., Cicchetti, D., \& Becker, B. (2000) The construct of resilience: a critical evaluation and guidelines for future work. Child Development, $71(3), 543-562$.

Madewell, A. N., \& Ponce-Garcia, E. (2016). Assessing resilience in emerging adulthood: The resilience scale (RS), Connor-Davidson resilience scale (CDRISC), and scale of protective factors (SPF). Personality and Individual Differences, 97, 249255. doi: 10.1016/j.paid.2016.03.036

Manzano-García, G., \& Ayala, G. C. (2013). Psychometric properties of Connor-Davidson Resilience Scale in a Spanish sample of entrepreneurs. Psicothema, 25(2), 245-251. doi: 10.7334/psicothema2012.183

McDonald, R. P., \& Ho, M. H. R. (2002). Principles and practice in reporting structural equation analyses. Psychological Methods, 7(1), 64-82. doi: 10.1037/1082-989X.7.1.64

Morales, M., \& González, A. (2014). ResilienciaAutoestima-Bienestar psicológico y Capacidad intelectual de estudiantes de cuarto medio de buen rendimiento de liceos vulnerables. Estudios pedagógicos (Valdivia), 40(1), 215-228. doi: 10.4067/S0718-07052014000100013

Muthén, L. K., \& Muthén, B. O. (1998-2015). Mplus User's guide (7th ed.). Los Angeles, CA: Muthén \& Muthén.

Palenzuela, D. (1983). Construcción y validación de una escala de autoeficacia percibida específica de situaciones académicas. Análisis y Modificación de Conducta, 9(21), 185- 219.

Pérez, A. M., Escobar, C. R., Toledo, M. R., Gutierrez, L. B., \& Reyes, G. M. (2018). Modelo de predicción de la deserción estudiantil de primer año en la Universidad Bernardo O' Higgins. Educação e Pesquisa, 44, e172094. doi: 10.1590/S16784634201844172094

Pidgeon, A. M., \& Pickett, L. (2017). Examining the differences between university students' levels of resilience on mindfulness, psychological distress and coping strategies. European Scientific Journal (Special Edition), 13(12), 103-113.

Ponce-Cisternas, F. P. (2015). Análisis exploratorio de modelos de ecuaciones estructurales sobre la escala Connor-Davidson (CD-RISC) en Chile y España. Salud y Sociedad, 6(3), 238-247. doi: $10.22199 / \mathrm{S} 07187475.2015 .0003 .00004$

Putnam, S. P., \& Rothbart, M. K. (2006). Development of short and very short forms of the Children's Behavior Questionnaire. Journal of Personality Assessment, 87(1), 102-112. doi: 10.1207/s15327752jpa8701_09

Reise, S. P., Scheines, R., Widaman, K. F., \&Haviland,
M. G. (2013). Multidimensionality and structural coefficient bias in structural equation modeling: A bifactor perspective. Educational and Psychological Measurement, 73(1), 5-26. doi: 10.1177/0013164412449831

Richardson, G. E. (2002). Metatheory of resilience and resiliency. Journal of Clinical Psychology, 58(3), 307-321.

Ríos-Risquez, M., Carrillo-García, C., \& Sabuco-Tebar, E. (2012). Resiliencia y Síndrome de Burnout en estudiantes de enfermería y su relación con variables sociodemográficas y de relación interpersonal. International Journal of Psychological Research, 5(1), 88-95.

Ríos-Risquez, M., García-Izquierdo, M., Sabuco-Tebar, E., Carrillo-Garcia, C., \& Martinez-Roche, M. (2016). An exploratory study of the relationship between resilience, academic burnout and psychological health in nursing students. Contemporary Nurse, 52(4), 430-439. doi: 10.1080/10376178.2016.1213648

Riveros, F., Bernal, L., Bohórquez, D., Vinaccia, S., \& Quiceno, J. (2017). Psychometric analysis of the Connor-Davidson Resilience Sacle (CD-RISC 10) in college students in Colombia. Psicología desde el Caribe, 34(3), 161-171.

Rodriguez, A., Reise, S. P., \& Haviland, M. G. (2016a). Evaluating bifactor models: calculating and interpreting statistical indices. Psychological Methods, 21(2), 137-150. doi: $10.1037 /$ met0000045

Rodriguez, A., Reise, S. P., \& Haviland, M. G. (2016b). Applying bifactor statistical indices in the evaluation of psychological measures. Journal of Personality Assessment, 98(3), 223-237.doi: 10.1080/00223891.2015.1089249

Sadat, M. (2009). Psychometric Evaluation of the Connor-Davidson Resilience Scale (CD-RISC) using Iranian students. International Journal of Testing, 9(1), 60-66. doi: 10.1080/15305050902733471

Salanova, M., Bresó, E., \& Schaufelli, W. (2005). Hacia un modelo espiral de las creencias de eficacia en el estudio de burnout y del engagement. Ansiedad y Estrés, 11, 215-231.

Sameroff, A. J., \& Rosenblum, K. L. (2006). Psychosocial constraints on the development of resilience. Annals of the New York Academy of Sciences, 1094(1), 116-124. doi: 10.1196/annals. 1376.010

Saris, W. E., Satorra, A., \& Van der Veld, W. M. (2009). Testing structural equation modeling or detection of misspecifications? Structural Equation Modeling, 16(4), 561-582. doi: 10.1080/10705510903203433

Sijtsma, K. (2009). On the use, the misuse, and the very limited usefulness of Cronbach's alpha. Psychometrika, 74, 107-120. doi: 10.1007/s11336- 
008-9101-0

Singh, K., \& Yu, X. (2010) Psychometric Evaluation of the Connor-Davidson Resilience Scale (CD-RISC) in a Sample of Indian Students, Journal of Psychology, 1(1), 23-30, doi: 10.1080/09764224.2010.11885442

Smits, I. A. M., Timmerman, M. E., Barelds, D. P. H., \& Meijer, R. R. (2015). The Dutch symptom checklist90-revised: is the use of the subscales justified? European Journal of Psychological Assessment, 31(4), 263-271. doi: 10.1027/1015-5759/a000233

Suárez-Montes, N., \& Diaz-Subieta, L. (2015). Estrés académico, deserción y estrategias de retención de estudiantes en la educación superior. Revista de Salud Pública, 17(2), 300-313. doi: 10.15446/rsap.v17n2.52891

Villalta, M. A. (2010). Factores de resiliencia asociados al rendimiento académico en estudiantes de contextos de alta vulnerabilidad social. Revista de Pedagogía, 31(88), 159-190.

Vizoso-Gómez, C., \& Arias-Gundín, O. (2018). Resiliencia, optimismo y burnout académico en estudiantes universitarios. European Journal of Education and Psychology, 11(1), 47-59. doi: 10.30552/ejep.v11i1.185

Watkins, M. W. (2018). Exploratory factor analysis: A guide to best practice. Journal of Black Psychology, 44(3), 219-246. doi: 10.1177/0095798418771807

West, S. G., Taylor, A. B., \& Wu, W. (2012). Model fit and model selection in structural equation modeling. En R. H. Hoyle (Ed.), Handbook of Structural Equation Modeling (pp. 209-231). New York, NY: Guilford.

Zinbarg, R. E., Yovel, I., Revelle, W., \& McDonald, R. P. (2006). Estimating generalizability to a latent variable common to all of a scale's indicators: A comparison of estimators for $\omega \mathrm{h}$. Applied Psychological Measurement, 30(2), 121-144. doi: $10.1177 / 0146621605278814 \mid$ 